\title{
Stratification and bundle structure of the set of conditioned invariant subspaces in the general case
}

\author{
J. Ferrer, F. Puerta, X. Puerta \\ Departament de Matemàtica Aplicada I \\ E.T.S. Enginyeria Industrial de Barcelona \\ Universitat Politècnica de Catalunya \\ Diagonal 647, 08028 Barcelona, Spain \\ E-mail: puerta@ma1.upc.es
}

Fax: 3434401937

\begin{abstract}
We extend some known results about the smooth stratification of the set of conditioned invariant subspaces for a general pair $(C, A) \in \mathbb{C}^{n} \times \mathbb{C}^{n+m}$ without any assumption on the observability. More precisely we prove that the set of $(C, A)$-conditioned invariant subspaces having a fixed BrunovskyKronecker structure is a submanifold of the corresponding Grassman manifold, with a vector bundle structure relating the observable and nonobservable part, and we compute its dimension. We also prove that the set of all $(C, A)$-conditioned invariant subspaces having a fixed dimension is connected, provided that the nonobservable part of $(C, A)$ has at most one eigenvalue (this condition is in general necessary).
\end{abstract}

Key words: Conditioned invariant subspace, Brunovsky basis, orbit space, fiber bundle, Grassmann manifold.

\section{Introduction}

The geometry and the structure of the set of $(C, A)$-conditioned invariant subspaces has been studied when $(C, A)$ is an observable pair by several authors, see for example $[\mathrm{HMP}],[\mathrm{FH}],[\mathrm{FPP}]$. The aim of this note is to extend the results of [FPP] to the case of a general pair $(C, A)$. More precisely, if $\operatorname{Inv}_{d}(C, A)$ denote the set of $d$-dimensional $(C, A)$-conditioned invariant subspaces we study the following three problems: (i) Smoothness. (ii) Dimension. (iii) Connectivity. For (i) and (ii) we consider both the real and complex case while for (iii) the field is assumed to be 


$$
\operatorname{Inv}_{d}(C, A)=\cup_{(M, F)} \operatorname{Inv}_{d}((C, A) ;(M, F))
$$

where $\operatorname{Inv}_{d}((C, A) ;(M, F))$ denote the set of $W \in \operatorname{Inv}_{d}(C, A)$ such that the restriction of $(C, A)$ to $W$ has a fixed Brunovsky form $(M, F)$ (see below for a precise definition), we obtain a finite smooth stratification of $\operatorname{Inv}_{d}(C, A)$. Moreover, we show that each stratum is a submanifold of the corresponding Grassmannian.

With regard to the problem (ii) we follow a quite different method of that in $[\mathrm{FPP}]$ in order to compute the dimension of $\operatorname{Inv}_{d}((C, A) ;(M, F)$. In fact, if the pair $(C, A)$ is observable, this dimension is obtained simply by counting parameters in a (nice) representation of each $W \in \operatorname{Inv}_{d}((C, A) ;(M, F))$ as a Toeplitz matrix. In our case and because of the presence of a Jordan part in the Brunovsky form of $(C, A)$, the above representation is much more involved and does not lead to an effective method of computing that dimension. We derive it by a reduction to the known cases. In fact, if we split $(C, A)$ into the observable and the nonobservable part, represented by a pair $\left(C_{o}, A_{o}\right)$ and an endomorphism $A_{\infty}$ respectively (and analogously with $(M, F)$ ), theorem 4.2 and proposition 4.4 relate the manifolds $\operatorname{Inv}_{d}((C, A),(M, F))$ and $\operatorname{Inv}_{d}\left(\left(C_{o}, A_{o}\right),\left(M_{o}, F_{o}\right)\right) \times \operatorname{Inv}_{d}\left(A_{\infty}, M_{\infty}\right)$ through a vector bundle structure which is not trivial in general as one might expect (see example 4.3). As an application of the theorem 4.2 we compute the dimension of $\operatorname{Inv}_{d}((C, A),(M, F))$ in terms of that of $\operatorname{Inv}_{d}\left(\left(C_{o}, A_{o}\right),\left(M_{o}, F_{o}\right)\right)$ and $\operatorname{Inv}_{d}\left(A_{\infty}, M_{\infty}\right)$ (theorem 4.6).

Finally, for the problem (iii) we extend the result on connectivity in [FPP] firstly to the set $\operatorname{Inv}_{d}((C, A))$ when $(C, A)$ is an observable pair making use again of the mentioned bundle structure (proposition 4.4) and next to the general case (theorem 4.11). We need for this case the connectivity of the set of invariant subspaces for a linear operator, $\operatorname{Inv}_{d}(A)$, and we remark that it can only be ensured in the case that $A$ has only one eigenvalue (if not, the eigenvectors corresponding to different eigenvalues span invariant rules laying in different connected components).

$\mathcal{M}_{p, q}$ will denote the set of complex matrices having $p$-rows and $q$-columns, and $\mathcal{M}_{p, q}^{*}$ the ones having maximal rank. If $p=q$, we will write simply $\mathcal{M}_{p}$ and $\mathcal{M}_{p}^{*}$ respectively. The latter, with the group structure of matrix multiplication, is the linear group $\mathrm{Gl}\left(\mathbb{C}^{p}\right)$.

If $X \in \mathcal{M}_{p, q},[X]$ will denote the subspace of $\mathbb{C}^{p}$ spanned by its columns. If $A \in \mathcal{M}_{p, q}$, we also denote by $A$ the linear map from $\mathbb{C}^{q}$ to $\mathbb{C}^{p}$ defined in a natural way by $A$.

If $E$ is a finite dimensional vector space and $F$ is a subspace of $E$, we say that a basis of $E$ is adapted to $F$ if it is obtained extending to $E$ a basis of $F$.

For any $\mathbb{C}$-vector space $\mathcal{Z}, \operatorname{Gr}_{d}(\mathcal{Z})$ will denote the Grassman manifold of $d$ dimensional subspaces of $\mathcal{Z}$. 
We first study the smoothness of the set of $d$-dimensional $(C, A)$-conditioned invariant subspaces for a fixed pair $(C, A) \in \mathcal{M}_{p, n} \times \mathcal{M}_{n}$ extending the corresponding results of the observable case. We denote this set by $\operatorname{Inv}_{d}(C, A)$ and we recall that a $d$-dimensional subspace $S$ of $\mathbb{C}^{n}$ is $(C, A)$-conditioned invariant (or $\left(\begin{array}{l}A \\ C\end{array}\right)$-invariant in [GLR]) if $A(S \cap \operatorname{ker} C) \subset S$. We remark that if $C$ is a zero-matrix $\operatorname{Inv}_{d}(C, A)$ is just the set of $A$-invariant subspaces of dimension $d$. In general it is not possible to endow $\operatorname{Inv}_{d}(C, A)$ with a differentiable structure; so, and analogously to [FPP] and $[\mathrm{S}]$, we define a finite partition of $\operatorname{Inv}_{d}(C, A)$ acording to the Brunovsky form of the restriction of $(C, A)$ to $S \in \operatorname{Inv}_{d}(C, A)$. In order to precise the meaning of this restriction we recall the following approach to the Brunovsky form.

We associate to the pair $(C, A)$ the linear map $f: \mathbb{C}^{n} \rightarrow \mathbb{C}^{n+p}$ defined by $f(x)=(A x, C x)$. We identify $\mathbb{C}^{n}$ with the subspace $\left\{(x, 0): x \in \mathbb{C}^{n}\right\}$ of $\mathbb{C}^{n+p}$. One can prove that two pairs $(C, A)$ and $\left(C^{\prime}, A^{\prime}\right)$ are Brunovsky equivalents (i.e. $\left(C^{\prime}, A^{\prime}\right)$ can be obtained from $(C, A)$ by an output injection and state space and output space change of variables) if and only if the corresponding linear maps $f$ and $f^{\prime}$ are related by $f^{\prime}=\left.\psi^{-1} f \psi\right|_{\mathbb{C}^{n}}$ being $\psi$ an automorphism of $\mathbb{C}^{n+p}$ making $\mathbb{C}^{n}$ invariant and $\left.\psi\right|_{\mathbb{C}^{n}}$ the automorphism of $\mathbb{C}^{n}$ defined by the restriction of $\psi$ to $\mathbb{C}^{n}$. Since $\psi$ can be interpreted as a change of basis of $\mathbb{C}^{n+p}$ adapted to $\mathbb{C}^{n}$, a canonical form of the matrix of $f$ with regard this change of bases characterize the Brunovsky class of $(C, A)$. In general one has the following proposition (see for example [FP]).

Proposition 2.1 Given a finite dimensional vector space $\mathfrak{X}$, a subspace $\mathcal{Y}$ of $\mathfrak{X}$ and a linear map $f: \mathcal{Y} \longrightarrow \mathfrak{X}$, there exist a maximal subspace $\mathcal{Y}_{\infty}$ of $\mathcal{Y}$ invariant by $f$ and bases $\left(B_{0}, B_{\infty}\right)$ of $\mathcal{Y}$ and $\left(B_{0}, B_{\infty}, B_{E}, B_{A}\right)$ of $\mathfrak{X}$ such that $B_{\infty}$ is a Jordan basis of $\mathcal{Y}_{\infty}$ with regard to $\left.f\right|_{\mathcal{Y}_{\infty}}, B_{0}=\left(v_{1} f\left(v_{1}\right), \ldots, f^{k_{1}-1}\left(v_{1}\right), \ldots, v_{r}, f\left(v_{r}\right), \ldots, f^{k_{r}-1}\left(v_{r}\right)\right)$, $B_{E}=\left(f^{k_{1}}\left(v_{1}\right), \ldots, f^{k_{r}}\left(v_{r}\right)\right.$ and $B_{A}$ arbitrary.

Definition 2.2 The bases $\left(B_{0}, B_{\infty}\right)$ and $\left(B_{0}, B_{\infty}, B_{E}, B_{A}\right)$ of the above proposition are called Brunovsky bases of $\mathcal{Y}$ and $\mathfrak{X}$ respectively. We will also refer to $v_{i}, f\left(v_{i}\right), \ldots, f^{k_{i}-1}\left(v_{i}\right)$ as a Brunovsky chain of the basis, to $v_{i}$ as the generator of the chain and to $k_{i}$ as the length of the chain, $1 \leq i \leq r$.

One can easily check that the matrix of $f$ with regard to Brunovsky bases is a Brunovsky matrix, this is to say, it has the form $\left(\begin{array}{l}N \\ E\end{array}\right)$, where $N=\operatorname{diag}\left\{N_{0}, N_{\infty}\right\}$, $E=\operatorname{diag}\left\{E_{0}, 0\right\}$, being $N_{\infty}$ a Jordan matrix and $\left(E_{0}, N_{0}\right)$ a Brunovsky observable pair, this is to say, $N_{0}=\operatorname{diag}\left\{N_{1}, \ldots, N_{r}\right\}$ each $N_{i}$ being the standard lower nilpotent $k_{i}$-matrix and $E_{0}=\operatorname{diag}\left\{E_{1}, \ldots, E_{r},\right\}$ each $E_{i}$ being a $k_{i}$-row matrix,

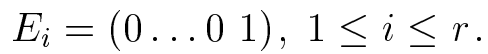

Identifying the pair $(C, A) \in \mathcal{M}_{p, n} \times M_{n}$, with the linear map $f$ defined above by $x \mapsto(A x, C x)$ we can apply the former proposition to conclude that there exist bases $\left(B_{0}, B_{\infty}\right)$ of $\mathbb{C}^{n}$ and $\left(B_{0}, B_{\infty}, B_{E}, B_{A}\right)$ of $\mathbb{C}^{n+p}$ such that the matrix of $f$ in these bases has a Brunovsky matrix $\left(\begin{array}{l}N \\ E\end{array}\right)$. Notice that the pair $(E, N)$ is Brunovsky equivalent to $(C, A)$. 
$f)$ to $S$ as the map $\left.f\right|_{S}: S \rightarrow \mathbb{C}^{n+p}$. If $S$ is $(C, A)$-conditioned invariant, we can associate to $\left.f\right|_{S}$ a pair of matrices by considering the matrix of $\left.f\right|_{S}$ with regard to any basis of $\mathbb{C}^{n+p}$ adapted to $S$, which has the form

$$
\left(\begin{array}{c}
\bar{A} \\
0 \\
\bar{C}
\end{array}\right)
$$

with $(\bar{C}, \bar{A}) \in \mathcal{M}_{p, d} \times \mathcal{M}_{d}$. Although $(\bar{C}, \bar{A})$ do not depend uniquely on $S$, its Brunovsky form does and is called the Brunovsky form of the restriction of $(C, A)$ to $S$.

Notice that $\left.f\right|_{S}$ represents (taking bases of $\mathbb{C}^{n+p}$ adapted to $S$ ) all the pairs Brunovsky equivalents to $(\bar{C}, \bar{A})$. So, it is natural to work with $f$ and $\left.f\right|_{S}$ rather than with the pairs $(C, A)$ and $(\bar{C}, \bar{A})$. For this reason we will write $\operatorname{Inv}_{d}(f)$ instead of $\operatorname{Inv}_{d}(C, A)$ and we will say that $S \in \operatorname{Inv}_{d}(f)$ is an $f$-conditioned invariant subspace.

Definition 2.3 With the above notation, we denote by $\operatorname{Inv}_{d}(f ;(M, F))$, or simple $\operatorname{Inv}(f ;(M, F))$ if no confusion is possible, the set of subspaces $S \in \operatorname{Inv}_{d}(f)$ such that the Brunovsky matrix of the restriction $\left.f\right|_{S}: S \longrightarrow \mathbb{C}^{n+p}$ is $\left(\begin{array}{c}M \\ F\end{array}\right)$.

We have that

$$
\operatorname{Inv}_{d}(f)=\bigcup_{M, F} \operatorname{Inv}(f ;(M, F)) .
$$

We will say that the Brunovsky matrix $\left(\begin{array}{c}M \\ F\end{array}\right)$ is compatible with $\left(\begin{array}{l}N \\ E\end{array}\right)$ if $\operatorname{Inv}(f ;(M, F))$ is nonempty. We will denote $\mathbb{C}^{n}$ by $\mathcal{Y}$ and $\mathbb{C}^{n+p}$ by $\mathfrak{X}$; we also will write $B-(\ldots)$ for Brunovsky-(...).

Remark 2.4 We recall that a B-matrix $\left(\begin{array}{c}M \\ F\end{array}\right)$ is compatible with $\left(\begin{array}{c}N \\ E\end{array}\right)$ if and only if the following conditions hold (see for example [1],[2]):

(a) $s \leq r$, and $h_{i} \leq k_{i}$ for $i=0,1,2, \ldots$

(b) the eigenvalues of $M_{\infty}$ are also eigenvalues of $N_{\infty}$, and for each one the corresponding Segre characteristics $\left(\eta_{1}(\lambda), \eta_{2}(\lambda), \ldots\right)$ and $\left(\varepsilon_{1}(\lambda), \varepsilon_{2}(\lambda), \ldots\right)$ verify: $\eta_{i}(\lambda) \leq \varepsilon_{i}(\lambda)$, for $i=1,2, \ldots$

Remark 2.5 It is known that the pair $(C, A)$ is observable if and only if the B-form of $\left(\begin{array}{l}A \\ C\end{array}\right)$ has not Jordan part $N_{\infty}$. So, it is natural to call a linear map $f: \mathcal{Y} \longrightarrow \mathfrak{X}$ as above observable if the B- matrix of $f$ has not Jordan part, this is to say, if $\mathcal{Y}_{\infty}=\{0\}$. Then, if $S$ is a subspace of $\mathcal{Y}$ the restriction $\left.f\right|_{S}: S \longrightarrow \mathfrak{X}$ is also observable. 
In this section we are going to endow the set $\operatorname{Inv}_{d}(f ;(M, F))$ with a differentiable structure through the identification of this set with an orbit space. This procedure is the same we have used in [FPP] (theorem 4.5) for the particular case where $f$ is assumed observable. In fact, the proof of that theorem does not make use of the observability of $f$ so that it works in the general case. Hence, we state the corresponding results without proof.

Let $\Phi$ be the map

$$
\Phi: \mathcal{M}_{n, d}^{*} \longrightarrow \operatorname{Gr}_{d}(\mathcal{Y})
$$

defined by $\Phi(X)=[X]$. For simplicity, we say that $X$ is a basis of $\Phi(X)$.

Theorem 3.1 With the above notation:

1. Let $\mathcal{S} \in \operatorname{Gr}_{d}(\mathcal{Y})$ such that $\left(\begin{array}{c}M \\ F\end{array}\right)$ is the $B$-matrix of the restriction $\hat{f}: \mathcal{S} \rightarrow \mathfrak{X}$. If $X$ is a $B$-basis of $\mathcal{S}$, then

(a) $N X=X M+N X F^{t} F$

(b) $E X=E X F^{t} F$

Moreover, $\mathcal{S}$ is $f$-invariant if and only if:

(c) EXFt has maximal rank.

2. Conversely, let $X \in \mathcal{M}_{n, d}^{*}$. If $X$ verifies the conditions (a), (b) and (c) above, then $\mathcal{S}=\Phi(X) \in \operatorname{Inv}(f ;(M, F))$.

This result motivates the following:

Definition 3.2 We denote by $\mathcal{M}((N, E) ;(M, F))$, or simply by $\mathcal{M}$ if no confusion is possible, the set of matrices $X \in \mathcal{M}_{n, d}^{*}$ which verify conditions (a), (b) and (c) in theorem 3.1.

Obviously, $\mathcal{M}$ is a submanifold of $\mathcal{M}_{n, d}^{*}$. In fact, it is an open subset of a linear subvariety of $\mathcal{M}_{n, d}$.

Then, from theorem 3.1 we have

Corollary 3.3 With the above notation,

$$
\Phi(\mathcal{M}((N, E) ;(M, F)))=\operatorname{Inv}(f ;(M, F)) .
$$

In general, $\Phi$ is not injective. In fact, we have that $\Phi(X)=\Phi\left(X^{\prime}\right)$ if and only if $X^{\prime}=X T$ for some $T \in \mathrm{Gl}\left(\mathbb{C}^{d}\right)$. If only matrices in $\mathcal{M}$ are considered, we have:

Proposition 3.4 Let $X, X^{\prime} \in \mathcal{M}$. Then, $\Phi(X)=\Phi\left(X^{\prime}\right)$ if and only if there is $T \in G l\left(\mathbb{C}^{d}\right)$ such that: $X^{\prime}=X T$, and 
$\left(b^{\prime}\right) F T=F T F^{t} F$

This proposition suggests the following definition:

Definition 3.5 We denote by $\mathcal{G}(M, F)$, or simply by $\mathcal{G}$ if no confusion is possible, the set of matrices $T \in G l\left(\mathbb{C}^{d}\right)$ which verify conditions ( $\left.a^{\prime}\right)$ and (b') in proposition 3.4.

Lemma 3.6 With the above notation, if $T \in \mathcal{G}$, then

(c') FTFt has maximal rank.

In fact, $\left(F T F^{t}\right)^{-1}=F T^{-1} F^{t}$.

Remark 3.7 Because of the last lemma, we can identify $\mathcal{G}$ with $\mathcal{M}((M, F) ;(M, F))$. However, we are mainly interested in the group structure of $\mathcal{G}$.

Lemma 3.8 With the above notation:

1. $\mathcal{G}$ is a subgroup of $G l\left(\mathbb{C}^{d}\right)$

2. $\mathcal{G}$ acts freely on $\mathcal{M}$ on the right by matrix multiplication.

Since $\mathcal{G}$ acts on $\mathcal{M}$ we can consider the orbit $X \mathcal{G}$ of an element $X \in \mathcal{M}$, which is the set $\{X T ; T \in \mathcal{G}\}$. Now a differentiable structure in $\operatorname{Inv}(f ;(M, F))$ can be defined by means of the following theorem:

Theorem 3.9 Let $\mathcal{M} / \mathcal{G}$ be the set of orbits under the action 3.8, and $\widetilde{\Phi}$ the map induced on it by $\Phi$. Then:

1. $\widetilde{\Phi}: \mathcal{M} / \mathcal{G} \longrightarrow \operatorname{Inv}(f ;(M, F))$ is a bijection.

2. The orbit space $\mathcal{M} / \mathcal{G}$ has a differentiable structure such that the natural projection $\pi: \mathcal{M} \longrightarrow \mathcal{M} / \mathcal{G}$ is a submersion.

Remark 3.10 In the conditions of the above theorem, it is known that the following properties are verified:

1. Each orbit $X \mathcal{G}=\{X T, T \in \mathcal{G}\}$ is a closed submanifold of $\mathcal{M}$, diffeomorphic to $\mathcal{G}$.

2. For any differentiable manifold $\mathcal{N}$, a map $\psi: \mathcal{M} / \mathcal{G} \longrightarrow \mathcal{N}$ is differentiable if and only if $\psi \circ \pi$ is differentiable. In particular, $\widetilde{\Phi}: \mathcal{M} / \mathcal{G} \longrightarrow \operatorname{Gr}_{d}(\mathcal{Y})$ is differentiable.

3. The submersion $\pi: \mathcal{M} \longrightarrow \mathcal{M} / \mathcal{G}$ is a principal bundle with structural group $\mathcal{G}$.

4. $\operatorname{dim}(\mathcal{M} / \mathcal{G})=\operatorname{dim} \mathcal{M}-\operatorname{dim} \mathcal{G}$.

Remark 3.11 We notice that all the results in this section hold also if we replace $\mathbb{C}$ for $\mathbb{R}$. 
In the last section we have introduced a differentiable structure in the set $\operatorname{Inv}_{d}(f ;(M, F))$ through the bijection between this set and the orbit space $\mathcal{M} / \mathcal{G}$. This, together with the fact that the natural projection $\pi: \mathcal{M} \longrightarrow \mathcal{M} / \mathcal{G}$ is a submersion will allow us to derive some differentiable and topological properties of $\operatorname{Inv}_{d}(f ;(M, F))$. We will make use of the existence of local sections of the submersion $\pi$. Let us recall that a local section is a differentiable map $\sigma: \mathcal{U} \longrightarrow \mathcal{M}$ where $\mathcal{U}$ is an open neighbourhood of $\mathcal{M} / \mathcal{G}$ such that $\pi \circ \sigma$ is the identity map. Then, for each $\mathcal{T} \in \mathcal{U}, \sigma(\mathcal{T})$ is a $B$-basis of $\mathcal{T}$. From theorem 4.1 until theorem 4.6 we can replace $\mathbb{C}$ by $\mathbb{R}$ in all the proofs and the results are therefore true in the real case. Nevertheless, the connectivity (propositions 4.7 and 4.8 and theorem 4.11) fails in the real case (see remark 4.13).

\subsection{Compatibility with the Grassmann Manifold}

Since we have the inclusion $\operatorname{Inv}_{d}(f ;(M, F)) \subset \operatorname{Gr}_{d}(\mathcal{Y})$ it is natural to ask if the differentiable structure of $\operatorname{Inv}_{d}(f ;(M, F))$ is the induced one from that of $\operatorname{Gr}_{d}(\mathcal{Y})$. The next theorem answer this question.

Theorem 4.1 $\operatorname{Inv}_{d}(f ;(M, F))$ with the differentiable structure defined by means of 3.9 is a submanifold of $G r_{d}(\mathcal{Y})$.

Proof. Because of the above identifications, this is equivalent to proving that $\mathcal{M} / \mathcal{G}$ is a submanifold of $\operatorname{Gr}_{d}\left(\mathbb{C}^{n}\right)=\mathcal{M}_{n, d}^{*} / \mathrm{Gl}\left(\mathbb{C}^{d}\right)$. For simplicity, we shall write $\mathcal{M}^{*} \equiv \mathcal{M}_{n, d}^{*}, G \equiv \mathrm{Gl}\left(\mathbb{C}^{d}\right)$. We consider the commutative diagram

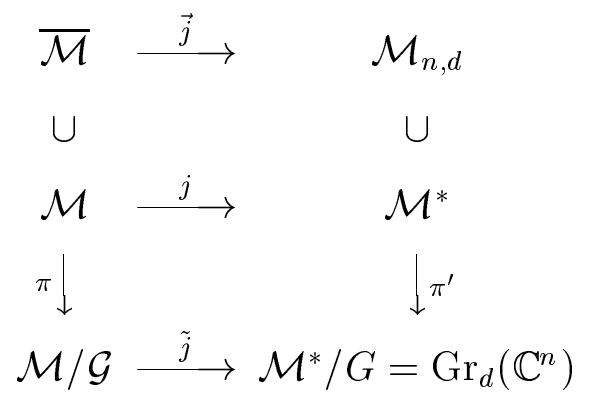

where $\pi$ and $\pi^{\prime}$ are the natural submersions, $\overline{\mathcal{M}}$ is the linear subspace of $\mathcal{M}_{n, d}$ defined by (a,b) of $3.1, j$ and $\vec{j}$ are the natural embeddings, and $\widetilde{j}$ is the injection (see (1) of 3.9) induced by $j$.

It is sufficient to obtain a local representation $\hat{j}=\sigma^{\prime} \circ \tilde{j} \circ \sigma^{-1}: \mathcal{U} \longrightarrow V$ of $\tilde{j}$, where $\sigma: \tilde{\mathcal{U}} \longrightarrow \mathcal{U}$ and $\sigma^{\prime}: \tilde{V} \longrightarrow V$ are local charts of $\mathcal{M} / \mathcal{G}$ and $\mathcal{M}^{*} / G$ respectively, such that $\mathcal{U}$ is a subspace of $V$, and $\widehat{j}$ is the natural inclusion. In fact, given $X \in \mathcal{M}$, we shall construct local charts at $\pi(X)$ and $\pi^{\prime}(X)$ of this kind as local sections of the submersions $\pi$ and $\pi^{\prime}$.

In order to that, let $L$ be the linear variety of $\overline{\mathcal{M}}$ ortogonal at $X$ to its $\mathcal{G}$-orbit $X \mathcal{G}=\pi^{-1}(\pi(X))$ (with regard to the usual hermitic product in $\mathcal{M}_{n, d}$ ). Then, there 
Next, if $X G=\pi^{\prime-1}\left(\pi^{\prime}(X)\right)$ is the $G$-orbit of $X$, it is clear (prop. 3.4) that $L \cap(X G)=\{X\}$, so that there is a linear variety $W$ of $\mathcal{M}_{n, d}$, minitransversal to $X G$ and containing $L$. Then, there are open neighbourhoods $V$ of $X$ in $W$ and $\tilde{V}$ of $\pi^{\prime}(X)$ in $\mathcal{M}^{*} / G$ such that the inverse of the diffeomorphism $\pi^{\prime}: V \longrightarrow \widetilde{V}$ is a local chart $\sigma^{\prime}$ for $\pi^{\prime}(X)$.

Finally, shrinking the neighbourhoods if necessary, it is clear that the embedding $\widehat{j}=\sigma^{\prime} \circ \widetilde{j} \circ \sigma^{-1}: \mathcal{U} \longrightarrow V$ represents the injection $\widetilde{j}: \widetilde{\mathcal{U}} \longrightarrow \widetilde{V}$, q.e.d..

\subsection{Bundle structure and dimension of $\operatorname{Inv}(f ;(M, F))$}

We recall that $N=\operatorname{diag}\left\{N_{0}, N_{\infty}\right\}$ and $E=\operatorname{diag}\left\{E_{0}, 0\right\}$. The matrices $N_{\infty}$ and $\left(\begin{array}{l}N_{0} \\ E_{0}\end{array}\right)$ can be interpreted as the matrices of the linear maps

$$
\begin{aligned}
& f_{\infty}: \mathcal{Y}_{\infty} \longrightarrow \mathcal{Y}_{\infty} \\
& \tilde{f}: \mathcal{Y} / \mathcal{Y}_{\infty} \longrightarrow \mathfrak{X} / \mathcal{Y}_{\infty}
\end{aligned}
$$

induced by $f$ in the natural way, with regard to the bases defined by the Brunovsky bases of $\mathcal{Y}$ and $\mathfrak{X}$.

Given $S \in \operatorname{Inv}(f ;(M, F))$, the maximal subspace of $S$ invariant by $f$ is $S_{\infty}=$ $S \cap \mathcal{Y}_{\infty}$. For any subspace $S_{0}$ such that $S=S_{\infty} \oplus S_{0}$, the restriction $\left.f\right|_{S_{0}}$ is observable. Moreover, if we split $(M, F)$ into the observable and nonobservable part analogously as $(N, E), M=\operatorname{diag}\left\{M_{0}, M_{\infty}\right\}$ and $F=\operatorname{diag}\left\{F_{0}, 0\right\}$ with $M_{0}=$ $\operatorname{diag}\left\{M_{1}, \ldots, M_{s}\right\}, E_{0}=\operatorname{diag}\left\{F_{1}, \ldots, F_{s}\right\}$ and $h_{\infty}=\operatorname{dim} M_{\infty}$, we have that $M_{\infty}$ is the Jordan matrix of $\left.f\right|_{S_{\infty}}$ while $\left(\begin{array}{c}M_{0} \\ F_{0}\end{array}\right)$ is the Brunovsky matrix of $\left.f\right|_{S_{0}}$.

Let $d_{0}=d-h_{\infty}=h_{1}+\ldots+h_{s}$ and $\operatorname{Inv}\left(f ;\left(M_{0}, F_{0}\right)\right)$ be the set of $d_{0}$-dimensional $(C, A)$-conditioned invariant subspaces such that the restriction of $f$ to them is observable with observability indices $h_{1}, \ldots, h_{s}$. Let $\operatorname{Inv}\left(f ; M_{\infty}\right)$ be the set $h_{\infty}$ dimensional invariant subspaces of $f$ such that the restriction of $f$ has Jordan form $M_{\infty}$. Then, For any $S_{0} \in \operatorname{Inv}\left(f ;\left(M_{0}, F_{0}\right)\right)$ and $S_{\infty} \in \operatorname{Inv}\left(f ; M_{\infty}\right)$, we have that $S_{0} \cap S_{\infty}=\{0\}$ and $f\left(S_{0} \oplus S_{\infty}\right) \cap \mathcal{Y} \subset S_{0} \oplus S_{\infty}$. Therefore $S \in \operatorname{Inv}(f ;(M, F))$. Notice that if $\left(B_{0}, B_{\infty}\right)$ is a $B$-basis of $S$, then $\left[B_{0}\right] \in \operatorname{Inv}\left(f ;\left(M_{0}, F_{0}\right)\right)$ and $\left[B_{\infty}\right] \in$ $\operatorname{Inv}\left(f ; M_{\infty}\right)$.

Then, we can state the main result of this section

Theorem 4.2 The map

$$
\begin{aligned}
& \theta: \operatorname{Inv}\left(f ;\left(M_{0}, F_{0}\right)\right) \times \operatorname{Inv}\left(f ; M_{\infty}\right) \longrightarrow \operatorname{Inv}(f ;(M, F)) \\
& \theta\left(\mathcal{S}_{0}, \mathcal{S}_{\infty}\right)=\mathcal{S}_{0} \oplus \mathcal{S}_{\infty}
\end{aligned}
$$

is a $\left(h_{\infty} s\right)$-dimensional vector bundle.

Proof. We will see that for any $\mathcal{S} \in \operatorname{Inv}(f ;(M, F))$ there is an open neighborhood $\mathcal{U}$ of $\mathcal{S}$, and a diffeomorphism

$$
\varphi: \mathcal{U} \times \mathcal{M}_{h_{\infty}, s} \longrightarrow \theta^{-1}(\mathcal{U}) \subset \operatorname{Inv}\left(f ;\left(M_{0}, F_{0}\right)\right) \times \operatorname{Inv}\left(f ; M_{\infty}\right)
$$


$\operatorname{Inv}(f ;(M, F))$. Thus, for each $\mathcal{T} \in \mathcal{U}, \sigma(\mathcal{T})$ is a $B$-basis of $\mathcal{T}$, of the form $\sigma(\mathcal{T})=$ $\left(\widehat{B}_{0}(\mathcal{T}), \widehat{B}_{\infty}(\mathcal{T})\right)$, so that $\left[\widehat{B}_{0}(\mathcal{T})\right] \in \operatorname{Inv}\left(f ;\left(M_{0}, F_{0}\right)\right)$ and $\left[\widehat{B}_{\infty}(\mathcal{T})\right] \in \operatorname{Inv}\left(f ; M_{\infty}\right)$, where $[(\ldots)]$ means the subspace spanned by $(\ldots)$. We denote by $u_{1}(\mathcal{T}), \ldots, u_{s}(\mathcal{T})$ the generators of the $B$-chains in $\widehat{B}_{0}(\mathcal{T})$, and by $e_{1}(\mathcal{T}), \ldots, e_{h_{\infty}}(\mathcal{T})$ the vectors of the basis $\widehat{B}_{\infty}(\mathcal{T})$.

Then, we define $\varphi$ as follows. Given $\mathcal{T} \in \mathcal{U}$ and $Z=\left(z_{j}^{i}\right) \in \mathcal{M}_{h_{\infty}, s}$, let $v_{j}(\mathcal{T})=u_{j}(\mathcal{T})+\sum_{1 \leq i \leq h_{\infty}} z_{j}^{i} e_{i}(\mathcal{T}), 1 \leq j \leq s$. It is trivial, that $v_{1}(\mathcal{T}), \ldots, v_{s}(\mathcal{T})$ generate linearly independent $B$-chains having the same length as those generated by $u_{1}(\mathcal{T}), \ldots, u_{s}(\mathcal{T})$ respectively; we denote them by $\widehat{B}_{0}(\mathcal{T})+Z \widehat{B}_{\infty}(\mathcal{T})$, that is to say: $\widehat{B}_{0}(\mathcal{T})+Z \widehat{B}_{\infty}(\mathcal{T})=\left\{v_{j}(\mathcal{T}), \widehat{f}\left(v_{j}(\mathcal{T})\right), \ldots, \hat{f}^{h_{j}-1}\left(v_{j}(\mathcal{T})\right) ; 1 \leq j \leq s\right\}$. Hence, $\left[\widehat{B}_{0}(\mathcal{T})+Z \widehat{B}_{\infty}(\mathcal{T})\right] \in \operatorname{Inv}\left(f ;\left(M_{0}, F_{0}\right)\right)$. Then, we define

$$
\varphi(\mathcal{T}, Z)=\left(\left[\widehat{B}_{0}(\mathcal{T})+Z \widehat{B}_{\infty}(\mathcal{T})\right],\left[\widehat{B}_{\infty}(\mathcal{T})\right]\right)
$$

Clearly, it is smooth, and

$$
(\theta \circ \varphi)(\mathcal{T}, Z)=\left[\widehat{B}_{0}(\mathcal{T})+Z \widehat{B}_{\infty}(\mathcal{T})\right] \oplus\left[\widehat{B}_{\infty}(\mathcal{T})\right]=\left[\widehat{B}_{0}(\mathcal{T})\right] \oplus\left[\widehat{B}_{\infty}(\mathcal{T})\right]=\mathcal{T}
$$

Also, it is easy to see that $\varphi$ is injective.

To show that $\varphi$ is surjective and that $\varphi^{-1}$ is smooth, we shall construct a local inverse. That is to say, given $\left(\mathcal{T}_{0}, \mathcal{T}_{\infty}\right) \in \theta^{-1}(\mathcal{U})$, we shall obtain a neighbourhood $V \subset \theta^{-1}(\mathcal{U})$ and a smooth map $\eta: V \longrightarrow \mathcal{U} \times \mathcal{M}_{h_{\infty}, s}$ such that $\varphi \circ \eta$ be the identity.

In order to do that, let $\sigma_{0}: \mathcal{U}_{0} \longrightarrow \mathcal{M}\left((N, E) ;\left(M_{0}, F_{0}\right)\right)$ and $\sigma_{\infty}: \mathcal{U}_{\infty} \longrightarrow$ $\mathcal{M}\left((N, E) ; M_{\infty}\right)$ be local sections at $\mathcal{T}_{0}$ and $\mathcal{T}_{\infty}$ of the respective submersions, so that for each $\mathcal{T}_{0}^{\prime} \in \mathcal{U}_{0}$ and for each $\mathcal{T}_{\infty}^{\prime} \in \mathcal{U}_{\infty}$, the images $\sigma_{0}\left(\mathcal{T}_{0}^{\prime}\right)$ and $\sigma_{\infty}\left(\mathcal{T}_{\infty}^{\prime}\right)$ are $B$-bases of $\mathcal{T}_{0}^{\prime}$ and $\mathcal{T}_{\infty}^{\prime}$ respectively.

We take $V=\left(\mathcal{U}_{0} \times \mathcal{U}_{\infty}\right) \cap \theta^{-1}(\mathcal{U})$, and we define the first component $\eta_{1}: V \longrightarrow \mathcal{U}$ of $\eta$ by means of: $\eta_{1}\left(\mathcal{T}_{0}^{\prime}, \mathcal{T}_{\infty}^{\prime}\right)=\theta\left(\mathcal{T}_{0}^{\prime}, \mathcal{T}_{\infty}^{\prime}\right)=\mathcal{T}_{0}^{\prime} \oplus \mathcal{T}_{\infty}^{\prime}$. Notice that we have two $B$-bases of $\mathcal{T}_{0}^{\prime} \oplus \mathcal{T}_{\infty}^{\prime}:\left(\sigma_{0}\left(\mathcal{T}_{0}^{\prime}\right), \sigma_{\infty}\left(\mathcal{T}_{\infty}^{\prime}\right)\right)$, and $\sigma\left(\mathcal{T}_{0}^{\prime} \oplus \mathcal{T}_{\infty}^{\prime}\right)=\left(\widehat{B}_{0}\left(\mathcal{T}_{0}^{\prime} \oplus \mathcal{T}_{\infty}^{\prime}\right), \widehat{B}_{\infty}\left(\mathcal{T}_{0}^{\prime} \oplus\right.\right.$ $\left.\mathcal{T}_{\infty}^{\prime}\right)$ ). Clearly, $\left[\widehat{B}_{\infty}\left(\mathcal{T}_{0}^{\prime} \oplus \mathcal{T}_{\infty}^{\prime}\right)\right]=\left[\sigma_{\infty}\left(\mathcal{T}_{\infty}^{\prime}\right)\right]=\mathcal{T}_{\infty}^{\prime}$. But, in general, $\left[\widehat{B}_{0}\left(\mathcal{T}_{0}^{\prime} \oplus\right.\right.$ $\left.\left.\mathcal{T}_{\infty}^{\prime}\right)\right] \neq\left[\sigma_{0}\left(\mathcal{T}_{0}^{\prime}\right)\right]=\mathcal{T}_{0}^{\prime}$. We will define $\eta_{2}\left(\mathcal{T}_{0}^{\prime}, \mathcal{T}_{\infty}^{\prime}\right)=Z \in \mathcal{M}_{h_{\infty}, s}$ in such a way that: $\left[\widehat{B}_{0}\left(\mathcal{T}_{0}^{\prime} \oplus \mathcal{T}_{\infty}^{\prime}\right)+Z \widehat{B}_{\infty}\left(\mathcal{T}_{0}^{\prime} \oplus \mathcal{T}_{\infty}^{\prime}\right)\right]=\mathcal{T}_{0}^{\prime}$. Then, the proof will be completed because: $(\varphi \circ \eta)\left(\mathcal{T}_{0}^{\prime}, \mathcal{T}_{\infty}^{\prime}\right)=\varphi\left(\mathcal{T}_{0}^{\prime} \oplus \mathcal{T}_{\infty}^{\prime}, Z\right)=\left(\left[\widehat{B}_{0}\left(\mathcal{T}_{0}^{\prime} \oplus \mathcal{T}_{\infty}^{\prime}\right)+Z \widehat{B}_{\infty}\left(\mathcal{T}_{0}^{\prime} \oplus \mathcal{T}_{\infty}^{\prime}\right)\right],\left[\widehat{B}_{\infty}\left(\mathcal{T}_{0}^{\prime} \oplus \mathcal{T}_{\infty}^{\prime}\right)\right]\right)=$ $\left(\mathcal{T}_{0}^{\prime}, \mathcal{T}_{\infty}^{\prime}\right)$

To obtain this $Z$, if $u_{1}, \ldots, u_{s}$ are now the generators of the $B$-chains in $\widehat{B}_{0}\left(\mathcal{T}_{0}^{\prime} \oplus\right.$ $\left.\mathcal{T}_{\infty}^{\prime}\right)$, we will determine $z_{1}, \ldots, z_{s} \in \mathcal{T}_{\infty}^{\prime}$ such that the subspace spanned by the $B$ chains generated by $u_{1}+z_{1}, \ldots, u_{s}+z_{s}$ be just $\mathcal{T}_{0}^{\prime}$. In order to do that, we shall consider separately the $B$-chains of different length. Let $v_{1}, \ldots, v_{s}$ be the generators of the $B$-chains in $\sigma_{0}\left(\mathcal{T}_{0}^{\prime}\right)$, and $\left(s_{1}, \ldots, s_{k_{1}}\right)$ the partition of $s$ according to the lengths $k_{1}, k_{1}-1, \ldots, 1$ of the corresponding $B$-chain, that is to say: $v_{1}, \ldots, v_{s_{1}}$ (and hence $u_{1}, \ldots, u_{s_{1}}$ ) generate $B$-chains having length $k_{1} ; v_{s_{1}+1}, \ldots, v_{s_{2}}$ (and hence $\left.u_{s_{1}+1}, \ldots, u_{s_{2}}\right)$ generate $B$-chains having length $k_{1}-1$; etc. If, on the other hand, we write $\mathcal{T}_{i}^{\prime}=\left(\mathcal{T}_{0}^{\prime} \oplus \mathcal{T}_{\infty}^{\prime}\right) \cap f^{-i}\left(\mathcal{T}_{0}^{\prime} \oplus \mathcal{T}_{\infty}^{\prime}\right), 1 \leq i \leq k_{1}$, we have:

$$
\mathcal{T}_{k_{1}}^{\prime}=\mathcal{T}_{\infty}^{\prime}
$$


$\mathcal{T}_{k_{1}-2}=\mathcal{J}_{\infty} \oplus\left[v_{1}, \ldots, v_{s_{1}}\right] \oplus\left[f\left(v_{1}\right), \ldots, f\left(v_{s_{1}}\right)\right] \oplus$

$$
\oplus\left[v_{s_{1}+1}, \ldots, v_{s_{2}}\right]
$$

etc.

and analogously for $u_{1}, \ldots, u_{s}$. Therefore, there are unique $z_{i} \in \mathcal{T}_{\infty}^{\prime}, 1 \leq i \leq s$, such that:

$$
\begin{aligned}
u_{i}+z_{i} & \in\left[v_{1}, \ldots, v_{s_{1}}\right], \quad 1 \leq i \leq s_{1} \\
u_{i}+z_{i} & \in\left[v_{1}, \ldots, v_{s_{1}}\right] \oplus\left[f\left(v_{1}\right), \ldots, f\left(v_{s_{1}}\right)\right] \oplus \\
& \oplus\left[v_{s_{1}+1}, \ldots, v_{s_{2}}\right], \quad s_{1}+1 \leq i \leq s_{2}
\end{aligned}
$$

etc.

Then, we define $\eta_{2}\left(\mathcal{T}_{0}^{\prime}, \mathcal{T}_{\infty}^{\prime}\right)=Z \in \mathcal{M}_{h_{\infty}, s}$ by means of: the columns of $Z$ are the coordinates of $z_{1}, \ldots, z_{s}$ in the basis $\widehat{B}_{\infty}\left(\mathcal{T}_{0}^{\prime} \oplus \mathcal{T}_{\infty}^{\prime}\right)$.

Clearly, they depend differentially on $\left(\mathcal{T}_{0}^{\prime}, \mathcal{T}_{\infty}^{\prime}\right)$. It remains to verify that

$$
\mathcal{T}_{0}^{\prime}=\left(\left[\widehat{B}_{0}\left(\mathcal{T}_{0}^{\prime} \oplus \mathcal{T}_{\infty}^{\prime}\right)+Z \widehat{B}_{\infty}\left(\mathcal{T}_{0}^{\prime} \oplus \mathcal{T}_{\infty}^{\prime}\right)\right]\right)
$$

We recall that $\mathcal{T}_{0}^{\prime}$ is spanned by the $B$-chains generated by $v_{1}, \ldots, v_{s}$, and that $\left[\widehat{B}_{0}\left(\mathcal{T}_{0}^{\prime} \oplus \mathcal{T}_{\infty}^{\prime}\right)+Z \widehat{B}_{\infty}\left(\mathcal{T}_{0}^{\prime} \oplus \mathcal{T}_{\infty}^{\prime}\right)\right]$ is spanned by the $B$-chains generated by $u_{1}+$ $z_{1}, \ldots, u_{s}+z_{s}$. Finally, by the construction of $z_{1}, \ldots, z_{s}$, we have:

$$
\begin{aligned}
& {\left[v_{1}, \ldots, v_{s_{1}}\right]=\left[u_{1}+z_{1}, \ldots, u_{s_{1}}+z_{s_{1}}\right]} \\
& {\left[v_{1}, \ldots, v_{s_{1}}\right] \oplus\left[f\left(v_{1}\right), \ldots, f\left(v_{s_{1}}\right)\right] \oplus\left[v_{s_{1}+1}, \ldots, v_{s_{2}}\right]=} \\
& =\left[u_{1}+z_{1}, \ldots, u_{s_{1}}+z_{s_{1}}\right] \oplus\left[f\left(u_{1}+z_{1}\right), \ldots, f\left(u_{s_{1}}+z_{s_{1}}\right)\right] \oplus \\
& \oplus\left[u_{s_{1}+1}+z_{s_{1}+1}, \ldots, u_{s_{2}}+z_{s_{2}}\right]
\end{aligned}
$$

etc.

The following example shows that in general $\theta$ is nontrivial.

Example 4.3 We switch for simplicity to the real case. Consider the Brunovsky pair

$$
(E, N)=\left(\left(\begin{array}{lll}
1 & 0 & 0 \\
0 & 1 & 0
\end{array}\right),\left(\begin{array}{lll}
0 & 0 & 0 \\
0 & 0 & 0 \\
0 & 0 & \lambda
\end{array}\right)\right) \lambda \in \mathbb{R}
$$

and the map $f$ defined by the matrix $\left(\begin{array}{l}N \\ E\end{array}\right)$. We fix the Brunovsky form for the restriction of $f$ to the 2-dimensional $f$-conditioned invariant subspaces to the pair

$$
(F, M)=\left(\left(\begin{array}{ll}
1 & 0 \\
0 & 0 \\
0 & 0
\end{array}\right),\left(\begin{array}{ll}
0 & 0 \\
0 & \lambda
\end{array}\right)\right)
$$




$$
M_{0}=0, \quad F_{0}=\left(\begin{array}{l}
1 \\
0 \\
0
\end{array}\right) \text { and } M_{\infty}=\lambda
$$

Notice that $\operatorname{Inv}_{1}\left(f ; M_{\infty}\right)=\left\{\left[e_{3}\right]\right\}$ and, since $f: \mathbb{R}^{3} \longrightarrow \mathbb{R}^{5}$ is such that $f(a, b, c)=$ $(0,0, \lambda c, a, b)$, the 1 -dimensional subspace $[x]$ with $x=a e_{1}+b e_{2}+c e_{3}$ belongs to $\operatorname{Inv}_{1}\left(f ;\left(F_{0}, M_{0}\right)\right)$ if and only if $a$ or $b$ are not zero. The vector bundle $\theta$ is defined in this case by

$$
\begin{aligned}
& \theta: \operatorname{Inv}_{1}\left(f ;\left(F_{0}, M_{0}\right)\right) \times \operatorname{Inv}_{1}\left(f ; M_{\infty}\right) \longrightarrow \operatorname{Inv}_{2}(f ;(M, F)) \\
& \theta\left([x],\left[e_{3}\right]\right)=[x] \oplus\left[e_{3}\right]
\end{aligned}
$$

Notice that $f\left([x] \oplus\left[e_{3}\right]\right) \cap \mathbb{R}^{3}=\left[e_{3}\right]$ and therefore $[x] \oplus\left[e_{3}\right]$ is $(F, M)$-conditioned invariant. On the other hand, we have that $\operatorname{Inv}_{2}(f ;(M, F))=\left\{\left[x, e_{3}\right] ; x=a e_{1}+\right.$ $b e_{2}+c e_{3}, a$ or $\left.b \neq 0\right\}$.

The fiber of $\theta$ has dimension 1 , and $\theta$ is not a trivial bundle as we show:

- $\operatorname{Inv}_{1}\left(f ; M_{0}\right)$ is the set of lines of $\mathbb{R}^{3}$ through the origin except the axe $\left[e_{3}\right]$, this is to say $\mathbb{P}_{2}(\mathbb{R})-\left\{\left[e_{3}\right]\right\}$ (a Moebius band). Moreover, since $\operatorname{Inv}_{1}\left(f ; M_{\infty}\right)=$ $\left\{\left[e_{3}\right]\right\}$, we have that $\operatorname{Inv}_{1}\left(f ;\left(F_{0}, M_{0}\right)\right) \times \operatorname{Inv}_{1}\left(f ; M_{\infty}\right)$ is homeomorphic to $\mathbb{P}_{2}(\mathbb{R})-\left\{\left[e_{3}\right]\right\}$

- $\operatorname{Inv}_{2}(f ;(F, M))$ is the set of plans containing the axe $\left[e_{3}\right]$ which can be identified with $\mathbb{P}_{1}(\mathbb{R})$

Therefore, $\theta$ is not trivial because $\mathbb{P}_{2}(\mathbb{R})-\left\{\left[e_{3}\right]\right\}$ is not homeomorphic to the cilinder $\mathbb{P}_{1}(\mathbb{R}) \times \mathbb{R}$.

From the above theorem we derive the following useful proposition.

Proposition 4.4 The map

$$
\begin{aligned}
& \psi: \operatorname{Inv}\left(f ;\left(M_{0}, F_{0}\right)\right) \longrightarrow \operatorname{Inv}\left(\tilde{f} ;\left(M_{0}, F_{0}\right)\right) \\
& \psi(\mathcal{S})=\left(\mathcal{S} \oplus \mathcal{Y}_{\infty}\right) / \mathcal{Y}_{\infty}
\end{aligned}
$$

is a $\left(k_{\infty} s\right)$-dimensional vector bundle.

Proof. Let consider Inv $(f ;(\bar{M}, \bar{F}))$, where $\bar{M}=\operatorname{diag}\left\{M_{0}, N_{\infty}\right\}, \bar{F}=\operatorname{diag}\left\{F_{0}, 0\right\}$.

Taking into account that $\operatorname{Inv}\left(f ; N_{\infty}\right)=\left\{\mathcal{Y}_{\infty}\right\}$, we can identify $\psi$ with the composition $\bar{\psi} \circ \bar{\theta}$ where

$$
\begin{aligned}
& \bar{\theta}: \operatorname{Inv}\left(f ;\left(M_{0}, F_{0}\right)\right) \times \operatorname{Inv}\left(f ; N_{\infty}\right) \longrightarrow \operatorname{Inv}(f ;(\bar{M}, \bar{F})) \\
& \bar{\theta}\left(\mathcal{S}, \mathcal{Y}_{\infty}\right)=\mathcal{S} \oplus \mathcal{Y}_{\infty} \\
& \bar{\psi}: \operatorname{Inv}(f ;(\bar{M}, \bar{F})) \longrightarrow \operatorname{Inv}\left(\widetilde{f} ;\left(M_{0}, F_{0}\right)\right) \\
& \bar{\psi}(\overline{\mathcal{S}})=\overline{\mathcal{S}} / \mathcal{Y}_{\infty}
\end{aligned}
$$


then $\overline{\mathcal{S}} \supset \mathcal{Y}_{\infty}$ and $\overline{\mathcal{S}} / \mathcal{Y}_{\infty} \in \operatorname{Inv}\left(\widetilde{f} ;\left(M_{0}, F_{0}\right)\right)$, and conversely. Therefore, $\bar{\psi}$ is the restriction of the diffeomorphism

$$
\Psi:\left\{\overline{\mathcal{S}} \in \operatorname{Gr}_{d+k_{\infty}}(\mathcal{Y}): \overline{\mathcal{S}} \supset \mathcal{Y}_{\infty}\right\} \longrightarrow \operatorname{Gr}_{d}\left(\mathcal{Y} / \mathcal{Y}_{\infty}\right), \Psi(\overline{\mathcal{S}})=\overline{\mathcal{S}} / \mathcal{Y}_{\infty}
$$

As an application we can compute the dimension of Inv $(f ;(M, F))$ in terms of that of $\operatorname{Inv}\left(\widetilde{f} ;\left(M_{0}, F_{0}\right)\right)$ and $\operatorname{dim} \operatorname{Inv}\left(f_{\infty} ; M_{\infty}\right)$. We first need the following remark

Remark 4.5 Since every subspace $S$ such that $f(S) \subset S$ is contained in $\mathcal{Y}_{\infty}$ one has that $\operatorname{Inv}\left(f ; M_{\infty}\right)=\operatorname{Inv}\left(f_{\infty} ; M_{\infty}\right)$.

Theorem 4.6 Let $f: \mathcal{Y} \longrightarrow \mathfrak{X}, M$ and $F$ be as in section 2. With the above notation:

$$
\begin{aligned}
\operatorname{dim} \operatorname{Inv}(f ;(M, F))= & \operatorname{dim} \operatorname{Inv}\left(\tilde{f} ;\left(M_{0}, F_{0}\right)\right)+\operatorname{dim} \operatorname{Inv}\left(f_{\infty} ; M_{\infty}\right)+\left(k_{\infty}-h_{\infty}\right) s= \\
= & \sum_{\substack{1 \leq i \leq n \\
1 \leq \leq \leq s}} \sup \left\{k_{i}-h_{j}+1,0\right\}-\sum_{1 \leq i, j \leq s} \sup \left\{h_{i}-h_{j}+1,0\right\}+ \\
& +\operatorname{dim} \operatorname{Inv}\left(f_{\infty} ; M_{\infty}\right)+\left(k_{\infty}-h_{\infty}\right) s
\end{aligned}
$$

Proof. To obtain the first equality it is sufficient to apply successively 4.2, 4.4 and 4.6:

$$
\begin{aligned}
\operatorname{dim} \operatorname{Inv}(f ;(M, F))= \\
=\operatorname{dim} \operatorname{Inv}\left(f ;\left(M_{0}, F_{0}\right)\right)+\operatorname{dim} \operatorname{Inv}\left(f ; M_{\infty}\right)-h_{\infty} s= \\
=\operatorname{dim} \operatorname{Inv}\left(\widetilde{f} ;\left(M_{0}, F_{0}\right)+k_{\infty} s+\right. \\
\quad+\operatorname{dim} \operatorname{Inv}\left(f_{\infty} ; M_{\infty}\right)-h_{\infty} s
\end{aligned}
$$

Then, the second one follows from 5.3 in [FPP].

Finally the following proposition gives another application of the theorem 4.2

Proposition 4.7 Each stratum $\operatorname{Inv}(f ;(M, F))$ is connected.

Proof. It follows from the theorem 4.2 taking into account the connectivity of $\operatorname{Inv}\left(\tilde{f} ;\left(M_{0}, F_{0}\right)\right)[\mathrm{FPP}]$ and Inv $\left(f_{\infty} ; M_{\infty}\right)([\mathrm{S}]$ and $[\mathrm{FPP}])$.

\subsection{Connectivity of $\operatorname{Inv}_{d}(f)$}

In [S] is proved that $\operatorname{Inv}_{d}(f)$ is connected when $f$ is an endomorphism having only one eigenvalue. We will generalize this result. Firstly, we consider the case when $f$ is observable.

Proposition 4.8 Let $f$ be observable. Then $\operatorname{Inv}_{d}(f)$ is connected. 
is also observable, so that its $B$-matrix is determined by its observability indices $h_{1} \geq \ldots \geq h_{s}$ (by convenience, we consider $h_{s+1}=\ldots=h_{r}=0$ ). Hence, we can note $\operatorname{Inv}_{d}(f ;(M, F)) \equiv \operatorname{Inv}_{s}(f ; h)$, and

$$
\operatorname{Inv}_{d}(f)=\cup_{h} \operatorname{Inv}_{d}(f ; h)
$$

where $h=\left(h_{1}, \ldots, h_{r}\right)$ runs over all the so-called "partitions of $d$ compatibles with $k=\left(k_{1}, \ldots, k_{r}\right)$ ", that is to say: $h_{1}+\ldots+h_{r}=d, h_{1} \geq \ldots \geq h_{r} \geq 0$, and $h_{i} \leq k_{i}$ for all $1 \leq i \leq r$.

We know that each stratum $\operatorname{Inv}_{d}(f ; h)$ is connected (prop. 4.7). In order to prove that the union of them is also connected, let consider the stratum $\operatorname{Inv}_{d}\left(f ; h_{*}\right)$ where $h_{*}$ is the only partition such that:

$$
h_{i} \geq h_{1}-1, \quad \text { if } \quad h_{i}<k_{i}
$$

It is sufficient to prove that, for any $h \neq h_{*}$ there is a (finite) sequence $h^{\prime}, h^{\prime \prime}, \ldots$ such that

$$
\operatorname{Inv}_{d}(f ; h) \cup \operatorname{Inv}_{d}\left(f ; h^{\prime}\right) \cup \operatorname{Inv}_{d}\left(f ; h^{\prime \prime}\right) \cup \ldots \cup \operatorname{Inv}_{d}\left(f ; h_{*}\right)
$$

is connected.

For that, given $h \neq h_{*}$ let be $\alpha<\beta$ such that

$$
\begin{aligned}
& h_{1}=h_{2}=\ldots=h_{\alpha}>h_{\alpha+1} \\
& \beta=\inf \left\{i: h_{i}<h_{1}-1, h_{i}<k_{i}\right\}
\end{aligned}
$$

Notice that $\alpha+1 \leq \beta \leq s+1$, and that if $\beta=s+1$, then $h_{\beta}=0$.

Then, we can consider $h^{\prime}$ defined by

$$
\begin{aligned}
& h_{\alpha}^{\prime}=h_{1}-1, \quad h_{\beta}^{\prime}=h_{\beta}+1 \\
& h_{i}^{\prime}=h_{i}, \quad \text { for any } i \neq \alpha, \beta
\end{aligned}
$$

The following lemma proves that

$$
\operatorname{Inv}_{d}(f ; h) \cup \operatorname{Inv}_{d}\left(f ; h^{\prime}\right)
$$

is connected. If $h^{\prime}=h_{*}$, the proof is finished. If not, we consider $h^{\prime \prime}$ in an analogous way, and so on.

Lemma 4.9 Let $h$ be a partition of $d$ compatible with $k$, such that, for some $1 \leq$ $\alpha<\beta \leq r$

$$
h_{\alpha}>h_{\beta}+1, \quad h_{\beta}<k_{\beta}
$$

There exist an $f$-conditioned invariant subspace $\mathcal{S} \subset \mathcal{Y}$ such that

$$
\mathcal{S} \in \operatorname{Inv}_{d}(f ; h) \cap \overline{\operatorname{Inv}_{d}\left(f ; h^{\prime}\right)}
$$

(where upperbar means "closure"). 
spanned by the chains

$$
v_{i}, f\left(v_{i}\right), \ldots, f^{h_{i}-1}\left(v_{i}\right) ; \quad 1 \leq i \leq s
$$

Clearly $\mathcal{S} \in \operatorname{Inv}_{d}(f ; h)$.

Now, let consider firstly the case $h_{\beta} \neq 0$. Then, let $v^{\prime}=f^{k_{\beta}-h_{\beta}-1}\left(w_{\beta}\right)$, so that $f\left(v^{\prime}\right)=v_{\beta}$; and, for each $\varepsilon>0$, let $\mathcal{S}_{\varepsilon} \subset \mathcal{Y}$ be the subspace spanned by the vectors

$$
\begin{aligned}
& v_{i}, f\left(v_{i}\right), \ldots, f^{h_{i}-1}\left(v_{i}\right) ; 1 \leq i \leq r, \quad i \neq \alpha, \beta \\
& f\left(v_{\alpha}\right), \ldots, f^{h_{\alpha}-1}\left(v_{\alpha}\right) \\
& v_{\beta}, \ldots, f^{h_{\beta}-1}\left(v_{\beta}\right) \\
& v_{\alpha}+\varepsilon v^{\prime}
\end{aligned}
$$

Notice that the last one spans a chain

$$
v_{\alpha}+\varepsilon v^{\prime}, f\left(v_{\alpha}\right)+\varepsilon v_{\beta}, \ldots, f^{h_{\beta}}\left(v_{\alpha}\right)+\varepsilon f^{h_{\beta}-1}\left(v_{\beta}\right)
$$

having length $h_{\beta}+1$, because of $f^{h_{\beta}+1}\left(v_{\alpha}\right) \in \mathcal{Y}$ (recall that $h_{\beta}+1<h_{\alpha}$, by hypothesis). Therefore, for any $\varepsilon>0$, we have $\mathcal{S}_{\varepsilon} \in \operatorname{Inv}_{d}\left(f ; h^{\prime}\right)$. And obviously, $\lim _{\varepsilon \rightarrow 0} \mathcal{S}_{\varepsilon}=\mathcal{S}$.

Finally, if $h_{\beta}=0$ the proof is analogous by considering $v^{\prime}=f^{k_{\beta}-1}\left(w_{\beta}\right)$ and $\mathcal{S}_{\varepsilon} \subset \mathcal{Y}$ the subspace spanned by the vectors

$$
\begin{aligned}
& v_{i}, f\left(v_{i}\right), \ldots, f^{h_{i}-1}\left(v_{i}\right) ; \quad 1 \leq i \leq r, \quad i \neq \alpha, \beta \\
& f\left(v_{\alpha}\right), \ldots, f^{h_{\alpha}-1}\left(v_{\alpha}\right) \\
& v_{\alpha}+\varepsilon v^{\prime}
\end{aligned}
$$

Remark 4.10 Notice that in the above proof, the hypothesis of $f$ being observable has been used only to ensure that any restriction $\widehat{f}: \mathcal{S} \longrightarrow \mathfrak{X}$ to an $f$-invariant subspace is also observable. Therefore, the proof can be easily generalized to prove that the union

$$
\bigcup_{M, F} \operatorname{Inv}_{d}(f ;(M, F))
$$

when $(M, F)$ runs over all the pairs of matrices such that $\left(\begin{array}{l}M \\ F\end{array}\right)$ is an observable $B$-matrix having $d$ columns. We will need this fact in the next theorem.

Finally, we have:

Theorem 4.11 Let $f: \mathcal{Y} \longrightarrow \mathfrak{X}$ be such that $f_{\infty}$ has only one eigenvalue. Then, $\operatorname{Inv}_{d}(f)$ is connected. 


$$
\operatorname{Inv}_{d}(f)=\bigcup_{M, F} \operatorname{Inv}_{d}(f ;(M, F))
$$

where $(M, F)$ runs over the set of pairs of matrices such that $\left(\begin{array}{c}M \\ F\end{array}\right)$ is a $B$-matrix having $d$ columns. We will denote this set by $B(d)$. Then, $M=\operatorname{diag}\left\{M_{0}, M_{\infty}\right\}$ and $F=\operatorname{diag}\left\{F_{0}, 0\right\}$, where $\left(\begin{array}{c}M_{0} \\ F_{0}\end{array}\right)$ is an observable $B$-matrix having $h_{0}$ columns, and $M_{\infty}$ is a Jordan $h_{\infty}$-square matrix with only one eigenvalue. If we denote these sets by $O B\left(h_{0}\right)$ and $\mathcal{J}\left(h_{\infty}\right)$ respectively we can identify

$$
B(d)=\bigcup_{\substack{h_{0}, h_{\infty} \\ h_{0}+h_{\infty}=d}}\left(O B\left(h_{0}\right) \times \mathcal{J}\left(h_{\infty}\right)\right)
$$

so that

$$
\operatorname{Inv}_{d}(f)=\bigcup_{h_{0}+h_{\infty}=d} \operatorname{Inv}_{h_{0}, h_{\infty}}(f)
$$

where

$$
\operatorname{Inv}_{h_{0}, h_{\infty}}(f) \equiv \bigcup_{O B\left(h_{0}\right) \times \mathcal{J}\left(h_{\infty}\right)} \operatorname{Inv}_{d}(f ;(M, F))
$$

Firstly, we will see that each one of these sets is connected, and afterwards that it is so the union of them.

For each $h_{0}, h_{\infty}$ fixed, with $h_{0}+h_{\infty}=d$, we have

$$
\begin{aligned}
\operatorname{Inv}_{h_{0}, h_{\infty}}(f) & =\underset{O B\left(h_{0}\right), \mathcal{J}\left(h_{\infty}\right)}{ } \theta\left(\operatorname{Inv}_{h_{0}}\left(f ;\left(M_{0}, F_{0}\right)\right) \times \operatorname{Inv}_{h_{\infty}}\left(f ; M_{\infty}\right)\right)= \\
= & \theta\left(\left[\bigcup_{O B\left(h_{0}\right)} \operatorname{Inv}_{h_{0}}\left(f ;\left(M_{0}, F_{0}\right)\right)\right] \times\left[\bigcup_{\mathcal{J}\left(h_{\infty}\right)} \operatorname{Inv}_{h_{\infty}}\left(f ; M_{\infty}\right)\right]\right)
\end{aligned}
$$

where $\theta$ is the map in (4.2). To see that this set is connected it is sufficient to see that both sets in the claudators are so. The first one is connected by (4.10). And the second one because of, from (4.4), we have

$$
\bigcup_{\mathcal{J}\left(h_{\infty}\right)} \operatorname{Inv}_{h_{\infty}}\left(f ; M_{\infty}\right)=\bigcup_{\mathcal{J}\left(h_{\infty}\right)} \operatorname{Inv}_{h_{\infty}}\left(f_{\infty}, M_{\infty}\right)=\operatorname{Inv}_{h_{\infty}}\left(f_{\infty}\right)
$$

and the later is connected (see $[\mathrm{S}]$ ). Finally, to see that the union

$$
\bigcup_{h_{0}+h_{\infty}=d} \operatorname{Inv}_{h_{0}, h_{\infty}}(f)
$$

is connected, it is sufficient to prove that, for any $h_{0}, h_{\infty}\left(0 \leq h_{0}<k_{0}, 0<h_{\infty} \leq\right.$ $\left.k_{\infty}\right)$ there is an $f$-invariant subspace $\mathcal{S}$ such that

$$
\mathcal{S} \in \operatorname{Inv}_{h_{0}, h_{\infty}}(f) \cap \overline{\operatorname{Inv}_{h_{0}+1, h_{\infty}-1}(f)}
$$

(where the upperbar means "closure"). In order to that, let be $k_{1} \geq \ldots \geq k_{r}$ the $B$-indices of $f, \lambda$ the unique eigenvalue of $f_{\infty}$, and $\delta_{1} \geq \ldots \geq \delta_{\rho}$ its Segre 
characteristic $\eta_{1} \geq \ldots \geq \eta_{\rho}$. Hence, we can note $\operatorname{Inv}_{d}(f ;(M, F)) \equiv \operatorname{Inv}_{d}(f ;(h, \eta))$, so that

$$
\operatorname{Inv}_{h_{0}, h_{\infty}}(f)=\bigcup_{h, \eta} \operatorname{Inv}(f ;(h, \eta))
$$

where $h$ runs over the partitions of $h_{0}$ compatible with $k$, and $\eta$ over the partitions of $h_{\infty}$ compatible with $\delta$. To end the proof of the theorem we prove the following lemma.

Lemma 4.12 Given $h_{0}, h_{\infty}$ with $0 \leq h_{0}<k_{0}, 0<h_{\infty} \leq k_{\infty}$, let be $h$ a partition of $h_{0}$ compatible with $k$ and $\alpha$ such that $h_{\alpha}<k_{\alpha}$, and let be $\eta$ a partition of $h_{\infty}$ compatible with $\delta$ and $\beta$ such that $\eta_{\beta}>0$. Then, let consider $h^{\prime}, \eta^{\prime}$ defined by

$$
\begin{array}{lll}
h_{\alpha}^{\prime}=h_{\alpha}+1, & h_{i}^{\prime}=h_{i} & \text { if } \quad i \neq \alpha \\
\eta_{\beta}^{\prime}=\eta_{\beta}-1, & \eta_{j}^{\prime}=\eta_{j} & \text { if } \quad j \neq \beta
\end{array}
$$

Then, there is an $f$ conditioned-invariant subspace $\mathcal{S}$ such that:

$$
\mathcal{S} \in \operatorname{Inv}(f ;(h, \eta)) \cap \overline{\operatorname{Inv}\left(f ;\left(h^{\prime}, \eta^{\prime}\right)\right)}
$$

(Hence: $\left.\mathcal{S} \in \operatorname{Inv}_{h_{0}, h_{\infty}}(f) \cap \overline{\operatorname{Inv}_{h_{0}+1, h_{\infty}-1}(f)}\right)$.

Proof. Let be $w_{i}, f\left(w_{i}\right), \ldots, f^{k_{i}-1}\left(w_{i}\right), 1 \leq i \leq r, B$-chains of $f$, and $w_{j},(f-$ $\lambda \operatorname{Id})\left(w_{j}\right), \ldots,(f-\lambda \mathrm{Id})^{\delta_{j}-1}\left(w_{j}\right), 1 \leq j \leq \rho$, a Jordan basis of $f_{\infty}$. Let consider

$$
\begin{array}{cc}
v_{i}=f^{k_{i}-h_{i}}\left(w_{i}\right) & 1 \leq i \leq s \\
\nu_{j}=(f-\lambda \mathrm{Id})^{\delta_{j}-\eta_{j}}\left(w_{j}\right) & 1 \leq j \leq \sigma
\end{array}
$$

where we assume that $h_{s}>h_{s+1}=0, \eta_{\sigma}>\eta_{\sigma+1}=0$. Let $\mathcal{S} \subset \mathcal{Y}$ the subspace spanned by

$$
\begin{array}{cl}
v_{i}, f\left(v_{i}\right), \ldots, f^{h_{i}-1}\left(v_{i}\right) & 1 \leq i \leq s \\
\nu_{j},(f-\lambda \mathrm{Id})\left(\nu_{j}\right), \ldots,(f-\lambda \mathrm{Id})^{\eta_{j}-1}\left(\nu_{j}\right) & 1 \leq j \leq \sigma
\end{array}
$$

Clearly $\mathcal{S} \in \operatorname{Inv}(f ;(h, \eta))$.

Now, let $u^{\prime}=f^{k_{\alpha}-h_{\alpha}-1}\left(w_{\alpha}\right)$, so that $f\left(u^{\prime}\right)=v_{\alpha}$. Finally, for any $\varepsilon>0$, let $\mathcal{S}_{\varepsilon}$ be the subspace spanned by

$$
\begin{array}{cl}
v_{i}, f\left(v_{i}\right), \ldots, f^{h_{i}-1}\left(v_{i}\right), & 1 \leq i \leq s \\
\nu_{j},(f-\lambda \mathrm{Id})\left(\nu_{j}\right), \ldots,(f-\lambda \mathrm{Id})^{\eta_{j}-1}\left(\nu_{j}\right), & 1 \leq j \leq \sigma, \quad j \neq \beta \\
\varepsilon u^{\prime}+\nu_{\beta},(f-\lambda \mathrm{Id}) \nu_{\beta}, \ldots,(f-\lambda \mathrm{Id})^{\eta_{\beta}-1}\left(\nu_{\beta}\right) &
\end{array}
$$

Obviously $\mathcal{S}=\lim _{\varepsilon \rightarrow 0} \mathcal{S}_{\varepsilon}$. And for all $\varepsilon>0, \mathcal{S}_{\varepsilon} \in \operatorname{Inv}\left(f ;\left(h^{\prime}, \eta^{\prime}\right)\right)$, because of $\varepsilon u^{\prime}+\nu_{\beta}$ spans a $B$-chain having length $h_{\alpha}+1$ :

$$
\varepsilon u^{\prime}+\nu_{\beta}, \varepsilon v_{\alpha}+f\left(\nu_{\beta}\right), \ldots, \varepsilon f^{h_{\alpha}-1}\left(v_{\alpha}\right)+f^{h_{\alpha}}\left(\nu_{\beta}\right)
$$

Applying the lemma 4.12 we have that each stratum is adherent to the maximal dimensional one and therefore the connectivity of $\operatorname{Inv}_{d}(f)$ is prooved. 
example the stratum defined by $k=(2)$ and $h=(1)$.

\section{References}

[BZ] I. Baragaña and I. Zaballa. Block Similarity Invariants of Restrictions to (A,B)-Invariants Subspaces. Linear Algebra and Appl., (220):31-62, 1995.

[CF] A. Compta-J. Ferrer. On $(A, B)^{t}$-invariant subspaces having extendible Brunovsky bases. Linear Alg. and Appl., (255):185-201, 1997.

[FP] J. Ferrer and F. Puerta. Similarity of Non-everywhere Defined Linear Maps. Linear Algebra and Appl, (168):27-55, 1992.

[FGP] J. Ferrer-M.I. García and F. Puerta. Differentiable Families of Subspaces. Linear Algebra and Appl., (199):229-252, 1994.

[FPP] J. Ferrer-F. Puerta-X. Puerta. Differentiable Structure of the Set of Controllable $(A, B)^{t}$-Invariant Subspaces. Linear Algebra and Appl., (275):161-177, 1998.

[FH] P.A. Fuhrmann, U. Helmke. A homeomorphism between observable pairs and conditioned invariant subspaces. Sys. and Contr, Letts, (30):217-223, 1997.

[GLR] I. Gohberg, P. Lancaster and L. Rodman. Invariant Subspaces of Matrices with Applications, Wiley, New York, 1986.

[HMP] D.Hinirichen, H.F.Münzner,D.Prätze-Wolters Parametrization of Conditioned Invariant Subspaces Systems and Control Letters,1,192-199, 1981

[S] M.A. Shayman. On the variety of invariant subspaces of a finite-dimensional linear operator. Trans. of AMS, (274.2):721-747, 1982.

[W] W.M. Wonham. Linear Multivariable Control: a Geometric Approach, Springer-Verlag, New York, 1979. 